\title{
Macular auto-fluorescence is a follow-up parameter for cystoids macular edema
}

\author{
ZHANG XinYuan $^{1 *}$, GONG XiaoHong ${ }^{2}$, WANG YanHong ${ }^{3} \&$ WANG NingLi ${ }^{1}$ \\ ${ }^{1}$ Beijing Institute of Ophthalmology, Beijing Tongren Eye Center, Tongren Hospital, Capital Medical University, Beijing 100730 China; \\ ${ }^{2}$ Beijing East Hospital, Beijing Chinese Traditional Medicine University, Beijing 100078, China; \\ ${ }^{3}$ Department of Epidemiology \& Biostatistics, School of Basic Medicine Peking Union Medical College \& Institute of Basic Medical Sciences, \\ Chinese Academy of Medical Sciences, Beijing 100005, China
}

Received March 26, 2015; accepted June 10, 2015; published online July 16, 2015

\begin{abstract}
This study aimed to evaluate if macular autofluorescence (MAF) is a valuable, non-invasive follow-up parameter for cystoid macular edema. A total of 71 eyes (71 cases) with cystoid macular edema (CME) were included in the study. Macular pigment (MP) was evaluated using HRA2 (infrared) IF and FA models. The density of MP was graded into three categories: without, partial, and normal amount of MP. A comparison was made between the baseline (before the first administration) level and at the fourth month, following three consecutive intravitreal lucentis injections every month. The morphology and distribution of MAF, and the density and distribution of MP were regarded as the main outcome measures. At the baseline visit, all eyes with CME had petaloid/irregular-shaped MAF in the macular area (100\%). No MAF was detected in the control eyes (0). There was significant difference in MAF between the CME and normal groups $(P=0.000)$. At the fourth monthly visit, normal levels of MP density without MAF was detected in 68 eyes (95.8\%) with the best corrected spectacular visual acuity increasing to at least 1 line accordingly. We conclude that macular MAF can be used as a follow-up parameter for patients with CME. MP and MAF can indirectly reflect the fovea cone function.
\end{abstract}

cystoid macular edema, macular autofluorescence, macular pigment

Citation: Zhang XY, Gong XH, Wang YH, Wang NL. Macular auto-fluorescence is a follow-up parameter for cystoids macular edema. Sci China Life Sci, 2015, 58: 773-777, doi: 10.1007/s11427-015-4905-5

Cystoid macular edema (CME), which is characterized by cystic retinal thickening or lipid deposition, still remains one of the major causes of blindness. It is prone to relapse following successful treatment. CME is a complex pathological process that has been demonstrated to be caused by multiple factors, including breakdown of the inner and outer blood-retinal barriers, oxidative stress, and elevated levels of vascular endothelial growth factor.

It still remains a challenge in clinical practice, not only because CME is difficult to treat, but also it is highly prone to relapse following successful treatment. New drugs, which

*Corresponding author (email: zhangxy1@ trhos.com) are administered via intravitreal injection, have emerged in recent years to become the first line treatment for diabetic macular edema (DME) that affects the central macula with loss of vision. Laser photocoagulation is still the gold standard of treatment for cases of CME that do not involve the central macular.

Intravitreal injection of anti-VEGF drugs have become the first line of treatment for CME that affects the central macula with loss of vision, however, due to its short effective duration, the drug needs to be injected monthly. It is necessary to have a non-invasive, routine method to detect the response of the treatments. Fundus fluorescein angiography (FFA) and optical coherence tomography (OCT) 
have been accepted as the gold standards for the diagnosis of macular edema [1]. Leakage of the micro-vessels in the macular eye, as well as the morphology of macular edema can be visualized with FFA. For DME, leaking microaneurysms contribute to foveal leakage in the early views, whereas the extent of the edema in the mid and late views can be demonstrated with FFA. However FFA is an invasive examination tool; the examination duration is longer, and it is difficult to use in clinical practice as a follow-up method, especially for elderly patients with poor general condition. Macular thickening, the shape of the sub-retinal spaces and the presence of vitreal traction can be observed by OCT, providing precise evaluation of retinal pathology in CME. However, the information of intuitive structure and overall morphology of ME is less evident [1-2].

In recent years, the autofluorescence (AF) technique has emerged and has been widely accepted as a non-invasive examination technique at the cellular level to observe retinal pigment epithelium (RPE) distribution [3]. The anatomo-pathologic correlations of AF findings have been established in animal studies [3]. Owing to the convenient inspection process, it has been widely applied in follow-up patients with several diseases such as age related macular degeneration [4], retinal pigmentosa [3], and Best disease [5]. In this study, we evaluate the correlation of AF and macular pigment density in patients with pre- and post- lucentis treated CME, and further aim to provide a non-invasive routine follow-up tool by studying the correlations of the macular pigment density and the morphology of macular edema.

\section{Study patients and inclusion criteria}

A total of 71 eyes (71 cases) with DME (16 eyes), CME secondary to central vein occlusion (CRVO, 30 eyes) and branch vein occlusion (BRVO, 23 eyes) Coat's disease (2 eyes), and non-infectious uveitis that received intravitreal injections of lucentis in Beijing Tongren Eye Center from 2012.8-2013.10 were included in this study. Enrolled in this study were 38 males and 33 females, aged 27-82 years old $(54.82 \pm 14.70)$ with a minimum follow-up of one year. The contralateral eyes or eyes from the age- and sex-matched normal subjects were used as the control.

All patients provided signed informed consent before intravitreal anti-VEGF treatment.

\subsection{Clinical examination and Diagnostic criteria}

Biomicroscopic features of CME included the presence of serous retinal detachment and disappearance of fovea light reflection. The diagnosis of CME was based on OCT findings, which are characterized by several dark cavities in the neuronal retina. FFA inclusion criteria includes petal-like hyperfluorescence in the late stages.
DME, CME secondary to BRVO, CRVO, non-infectious uveitis and Coat's disease were diagnosed using the patient's medical history, indirect ophthalmoscopy, mydriatic fundus photography and fluorescein fundus angiography (FFA).

The best corrected spectacular visual acuity (BSVA) was measured using standard Chinese $\log$ MAR acuity charts. Changed BSVA was defined as a loss or gain of more than $10 \log$ MAR letters; otherwise, it was defined as unchanged. FFA was performed using the Heidelberg HRA2 fundus angiography system.

Eyes were followed up by BSVA, AF, MP and OCT every month.

\subsection{Intravitreal injection of Lucentis}

Eyes in which macular edema was determined to be $>250$ $\mu \mathrm{m}$ by OCT and in which the duration of CME was at least $>2$ months received intravitreal injections of lucentis. The treatment strategy was three plus P.R.N during the first year of follow-up. Patients were asked to follow up every month. The intravitreal injection protocol was described previously [5].

\subsection{Detection of auto-fluorescence and macular pigment}

MAF and MP were recorded with a confocal laser scanning laser ophthalmoscope (Retinal Angiograph 2; Heidelberg Engineering, Heidelberg, Germany). The high-resolution image field was set to: view $55^{\circ}$ and $1536 \times 1536$ pixels. The pupil was dilated to a diameter of $>6 \mathrm{~mm}$ for MAF and MP detection. Fluorescent angiography (FA mode without fluorescein injection, excitation wavelength $488 \mathrm{~nm}$ ) mode and infrared (IR mode, wavelength $830 \mathrm{~nm}$, intensity of 100\%) light was used to detect MP. FA (without injection of fluorescein) was applied for detection of macular MAF.

\subsection{Grading of macular pigment and MAF}

According to Zhang et al. [6], macular pigment is graded into three categories: those without foveal MP, those with partial pigment and those with normal amounts of MP. These categories were compared with auto-fluorescence and OCT. Auto-fluorescence was graded into two categories: exist and non-exist.

\subsection{Statistical analysis}

All data are presented as means \pm standard error of the mean (SEM). Data analysis was performed with the GraphPad Prism (version 4.0, San Diego, CA, USA). The Fisher Exact Test was used to compare the continued variance for CME and the normal control groups. The Monte Carlo exact test was used to find out the correlations between the two vari- 
ance MP and MAF, Linear-by-Linear association test was used to test the two variance to explain if there is a linear trend, $P<0.05$ was considered statistically different.

\section{Results}

\subsection{Auto-fluorescence in the macular}

At the baseline visit, all 71 cases of CME showed petal-like or irregular fluorescence images in FFA. The petal-like or irregular appearance and the location of the lesion were detected by MAF accordingly (Figure 1). However, MAF is not correlated with the patient's age, sex and type of the primary diseases (BRVO, CRVO, diabetic retinopathy or Coat's disease). MAF was not detected in the normal eyes. At the fourth monthly visit, MAF was detected in 2 eyes $(2.8 \%)$. There is a significant difference between the two groups $(P=0.000)$.

\subsection{Macular pigment density in normal and study eyes}

At the baseline visit, MP was not detected in any of the study eyes; however, normal amounts of MP were detected in $100 \%$ of the control normal eyes. At the fourth monthly follow-up visit, the percentage of normal amount of MP, partial MP and without MP was 68 (95.8\%), 2 (2.8\%), 1 (1.4\%) respectively in the CME group; normal amount MP was detected in all the control eyes (Figure 2) there is a significant difference in the two groups $(P=0.000)$. MP is not correlated with the patient's age, sex and type of the prima- ry diseases (BRVO, CRVO, diabetic retinopathy or coat's disease)

A Linear-by-Linear Association test revealed that there is linear trend between the two variables (Table 1). MP was negatively correlated with MAF in this study $\left(\chi^{2}=42.123\right.$, $P=0.000$ ). The Kappa coefficient is 1.00 .

\subsection{Correlations of MAF, MP and foveal cone function}

A total of 22 eyes $(30.98 \%)$ with a BSVA of $20 / 200$ or worse had no MP in the fovea, but MAF could be detected accordingly. Furthermore, 3 eyes $(4.23 \%)$ with BSVA of 20/40 or better had normal amounts of MP but MAF could not be detected. At the fourth monthly visit, normal amounts of MP density without MAF was found in 19 eyes

Table 1 The correlation of fundus auto-fluorescence and macular pigment density in cystoid macular edema ${ }^{\text {a) }}$

\begin{tabular}{ccc}
\hline & Baseline N $(\%)$ & 4 Month visit N $(\%)$ \\
\hline Grading of MP & & \\
Normal & $0(0 \%)$ & $2(2.82 \%)$ \\
Partial & $0(0 \%)$ & $69(97.18 \%)$ \\
No MP & $71(100 \%)$ & $0(0.00)$ \\
MAF & & \\
Detectable signal & $71(100 \%)$ & $0(0 \%)$ \\
Reduced signal & $0(0 \%)$ & $69(97.18 \%)$ \\
Not detectable & $0(0 \%)$ & $2(2.82 \%)$ \\
\hline
\end{tabular}

a) Kappa coefficient (to measure the agreement between two methods in one sample): 1.00; MP: macular pigment, MAF: macular autofluorescence
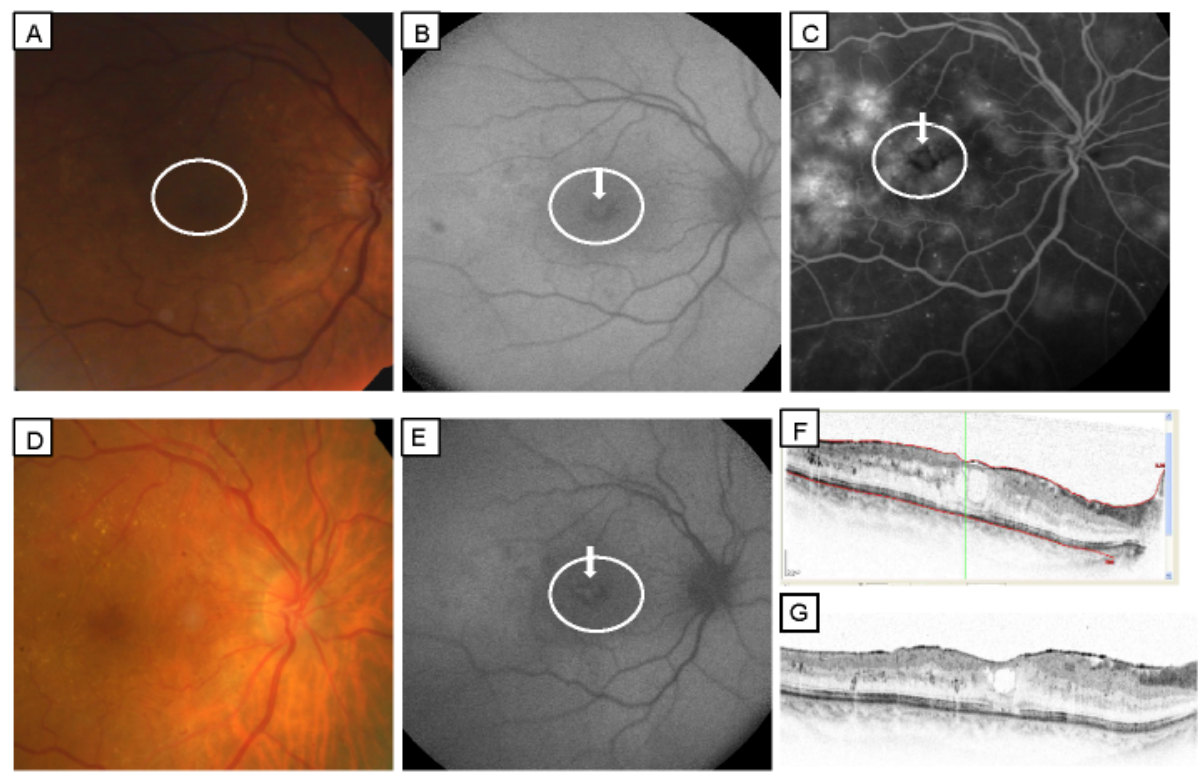

Figure 1 Color fundus photo of a right eye with diabetic macular edema (A). Macular autofluorescence of the eye (B) and late angiographic image (C) the performance in the fovea consistent with round shape of high fluorescence (white arrows) which is surround by dark macular pigment. Color fundus photo of the same eye after three intravitreal injections of Lucentis (D). The signal of the hyperflorence which is surrounded by irregular dark macular pigment is reduced compared with the eye post-intravitral injection of lucentis (E) (white arrow). OCT (D) shows cystoid macular changes and retinal thickening in the right eye (the topographic maps of retinal thickness pre-operation $(424 \mu \mathrm{m})$ and post-operation $(320 \mu \mathrm{m},(\mathrm{F}))$. 

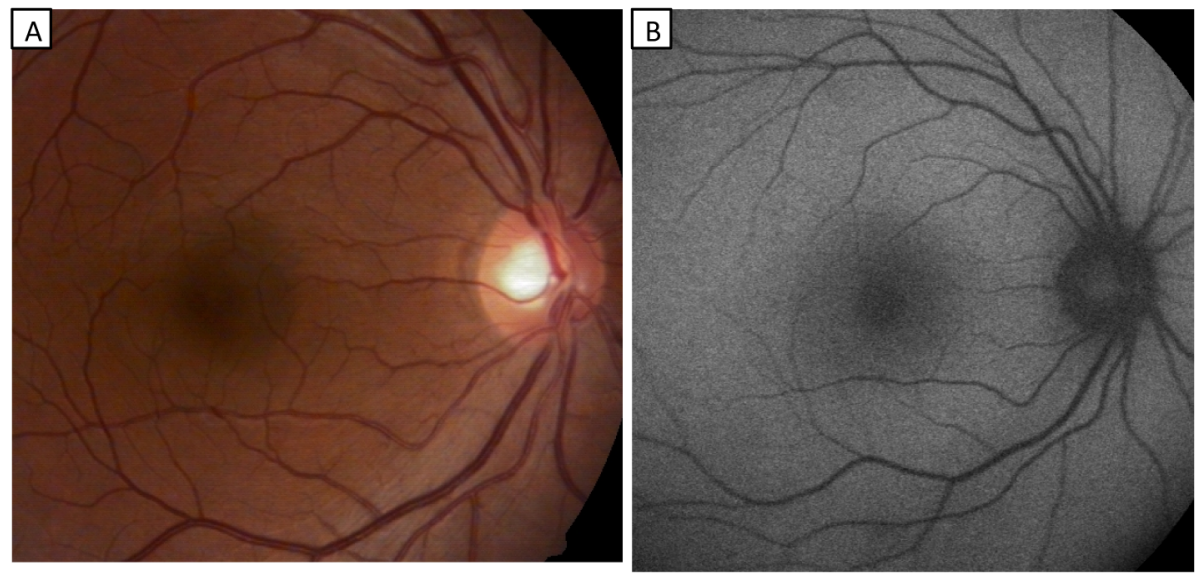

C

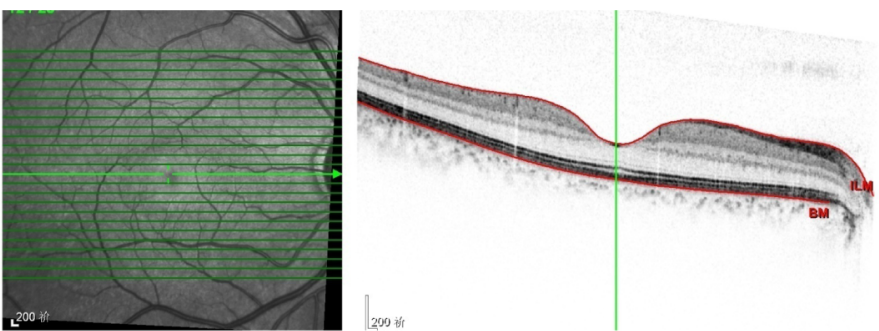

Figure 2 Color fundus image of a normal eye (A) macular area presents a complete macular pigment without detectable macular autoflorence (B, white arrow). OCT showed normal retinal layers of the structure (C).

(26.77\%), and 11 eyes (15.49\%) has no MP in the fovea with the BSVA of 20/200 or worse (Table 2 and 3). The correlations of BSVA between pre- and post-intravitreal injections of lucentis is summarized in Figure 3.

A significant difference in BSVA- and MP-MAF was found between pre- and post-operation groups $(P 1=0.000$, $P 2=0.000)$.

\section{Discussion}

In this study, we evaluated the correlation between MAF and the MP density pre- and post- lucentis intravitreal injection. The findings indicate that MAF is a rapid, noninvasive technique that may provide new insights in the follow up of DME.

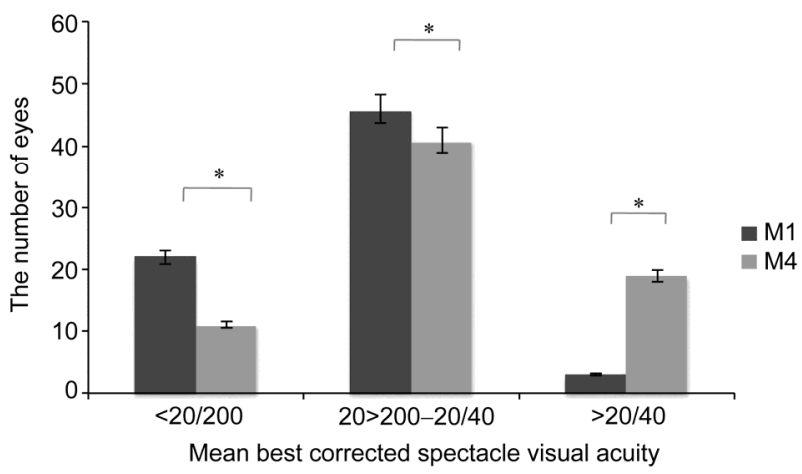

Figure 3 The correlations of BCSA between pre and post IV injections. BCSA: the best corrected spectacle visual acuity, IV: Intravitreal injection. $*: P<0.05$.
Table 2 Correlations of MAF, MP and foveal cone function (A Baseline visit (Month 1)) $)^{\mathrm{a}}$

\begin{tabular}{cccc}
\hline & $20 / 200$ or worse & $20 / 200 \sim 20 / 40$ & $20 / 40$ or better \\
\hline Grading of MP & & & \\
Normal & 0 & & $3(100 \%)$ \\
Partial & 0 & $2(4.35 \%)$ & 0 \\
No MP & $22(100 \%)$ & $44(95.65 \%)$ & 0 \\
Total & $22(100 \%)$ & $46(100 \%)$ & $3(100 \%)$ \\
$P$ value & 0.0000 & 0.0000 & 0.0000 \\
MAF & & & \\
Petal-like MAF & $22(100 \%)$ & $44(95.65 \%)$ & \\
Reduced signal & & $2(4.35 \%)$ & $3(100 \%)$ \\
Not detectable & 0 & 0 & $3(100 \%)$ \\
Total & $22(100 \%)$ & & 0.0000 \\
$P$ value & 0.0000 & 0.0000 & \\
\hline
\end{tabular}

a) MAF: macular auto-fluorecence, MP: macular

Table 3 Correlations of MAF, MP and foveal cone function (Month 4)

\begin{tabular}{cccc}
\hline & $20 / 200$ or worse & $20 / 200-20 / 40$ & $20 / 40$ or better \\
\hline Grading of MP & & & \\
Normal & 0 & $2(4.35 \%)$ & $19(100 \%)$ \\
Partial & 0 & $4(95.65 \%)$ & 0 \\
No MP & $11(100 \%)$ & $41(100 \%)$ & $19(100 \%)$ \\
Total & $11(100 \%)$ & 0.0000 & 0.0000 \\
$P$ value & 0.0000 & & \\
MAF & & $44(95.65 \%)$ & \\
Petal-like MAF & $11(100 \%)$ & 0 & $3(100 \%)$ \\
Reduced signal & 0 & $2(4.35 \%)$ & $3(100 \%)$ \\
Not detectable & 0 & & 0.0000 \\
Total & $22(100 \%)$ & 0.0000 & \\
$P$ value & 0.0000 & &
\end{tabular}

a) MAF: macular auto-fluorecence, MP: macular 
Dysfunction of cones and photoreceptors is highly correlated with edema and cysts formation. In CME, fluid accumulates in the extracellular space of the outer plexiform and inner nuclear layers, cyst formation occurs due to the radial distribution of Henle fibers in the outer plexiform layer [7]. Directional sensitivity and visual pigment density have been demonstrated in eyes with CME [6].

Current effective intervention for CME includes laser photocoagulation, surgery and drugs. Anti-VEGF therapy has been the first-line treatment for CME. Since the effective-time of anti-VEGF treatment is shorter within a month, a repeated treatment strategy is applied for most patients with CME, a routine non-invasive and effective examination to follow up post treat CME patients is necessary.

In our previous pilot study (which is a small case serial study), it was demonstrated that MP density is highly correlated with the presence of MAF [2]. One of the main functions of MP is a "filtration" material which [8] can absorb shorter wavelength light such as blue light (absorption range of $400 \sim 540 \mathrm{~nm}$, peak $460 \mathrm{~nm}$ ) and protect the underneath cones. In normal eyes, blockage and presence of normal amounts of MP and macular MAF were hardly detected, but in some pathological conditions, reduction of the density of MP, could lead to transmission of the blue excitation, and MAF can thus be detected [9]. Formation of cysts within the retina in the cystoid macular edema, resulting in the displacement of MP through the RPE layer, causes the petal-like appearance of the MAF, suggesting that MAF and MP density is negatively correlated. There have been two hypotheses to explain the hypo-AF in CME: 1) temporary displacement of MP caused by the cysts, MAF was considered to be "pseudo" or "relative" AF [10] 2) the presence of some fluorophores inside the cysts. This hypothesis was puts forward as an explanation for the correlations in which MP density is not correlated with detectable MAF.

Our previous study demonstrated that MP density was highly corrected with foveal cone functions. In this study, we also found that when MP density can be detected following intravitreal injection of anti-VEGF therapy, the visual acuity improved and MAF was accordingly nondetectable in the treated eyes. Furthermore, it was interesting to discover that in eyes with BSVA below 20/200, no MP could be detected but MAF could, supporting the idea that MP and MAF are indicators for fovea cone function. A well designed clinical trial is warranted to further to quantitatively study the relationship of fovea cone function and MP or MAF.
The results of this study revealed that MAF and MP have diagnostic values in the study of this disease and can be utilized as follow-up parameters for a variety of diseases with CME. The monitoring and prognostic function of MAF, provides a more valuable clinical technical parameter. In our previous study, we confirmed that MP is an indicator of macular cone function [6]. The negative correlation of MP and MAF also demonstrates that AF indicates macular cone function, warranting a relatively large sample of clinical trials for validation. Combining the two techniques of OCT and MAF could provide ideal follow-up information for patients with CME.

This work was partly supported by the National Natural Science Foundation of China (81170859), Beijing Municipal Education Commission Key Project (KZ201210025027) and Beijing Science and Technology New Star Project (2004B28).

1 Antcliff RJ, Stanford MR, Chauhan DS,Chauhan DS, Graham EM, Spalton DJ, Shilling JS, Ffytche TJ. Comparison between optical coherence tomography and fundus fluorescein angiography for the detection of cystoid macular edema in patients with uveitis. Ophthalmology, 2000, 107: 593-599

2 Zhang X, Gillies M, Zhu X, Wang X, Xiao Y, Xu X. The correlation study of mauclar autofluorescence and macular pigment in cystoid macular edema. Ophthalmol CHN, 2010, 19: 357-360

3 Aizawa S, Mitamura Y, Hagiwara A, Sugawara T, Yamamoto S. Changes of fundus autofluorescence, photoreceptor inner and outer segment junction line, and visual function in patients with retinitis pigmentosa. Clin Experiment Ophthalmol, 2010, 38: 597-604

4 Bearelly S, Cousins SW. Fundus autofluorescence imaging in age-related macular degeneration and geographic atrophy. Adv Exp Med Biol, 2010, 664: 395-402

5 Jarc-Vidmar M, Kraut A, Hawlina M. Fundus autofluorescence imaging in Best's vitelliform dystrophy. Klin Monbl Augenheilkd, 2003, 220: $861-867$

6 Zhang X, Hargitai J, Tammur J, Amy H, Rando A, Stanley C, Gouras P. Macular pigment and visual acuity in Stargardt macular dystrophy. Graefes Arch Clin Exp Ophthalmol, 2002, 240: 802-809

7 Zhang X, Zeng H, Bao S, Wang N, Gillies M. Diabetic macular edema: new concepts in patho-physiology and treatment. Cell Biosci, 2014, 4: 27

8 Rapp LM, Maple SS, Choi JH. Lutein and zeaxanthin concentrations in rod outer segment membranes from perifoveal and peripheral human retina. Invest Ophthalmol Vis Sci, 2000, 41: 1200-1209

9 Snodderly DM, Brown PK, Delori FC, Auran JD. The macular pigment. I. Absorbance spectra, localization, and discrimination from other yellow pigments in primate retinas. Invest Ophthalmol Vis Sci, 1984, 25: 660-673

10 Snodderly DM, Auran JD, Delori FC. The macular pigment. II. Spatial distribution in primate retinas. Invest Ophthalmol Vis Sci, 1984, 25: 674-685

Open Access This article is distributed under the terms of the Creative Commons Attribution License which permits any use, distribution, and reproduction in any medium, provided the original author(s) and source are credited. 